\title{
Temperature Insensitive Polarization Maintaining Photonic Crystal Fiber Refractive Index Sensor
}

\author{
Xuan Guo \\ College of Information Science and Engineering \\ Yanshan University \\ Qinhuangdao, P. R. China \\ The Key Laboratory for Special Fiber and Fiber Sensor \\ of Herbei Province \\ Yanshan University \\ Qinhuangdao, P.R. China \\ e-mail: guoxuan@ysu.edu.cn \\ Feng Liu \\ College of Information Science and Engineering \\ Yanshan University \\ Qinhuangdao, P. R. China \\ The Key Laboratory for Special Fiber and Fiber Sensor \\ of Herbei Province \\ Yanshan University \\ Qinhuangdao, P. R. China \\ e-mail: kennan@ysu.edu.cn
}

\begin{abstract}
A tapered polarization maintaining photonic crystal fiber based on dual-mode interference is proposed for measuring liquid refractive index. The sensing principle is based on the interference between $L P_{01}$ and $L P_{11}$ modes in the photonic crystal fiber. The output intensity distribution of the two modes in the fiber is investigated in detail. It is shown that when the external refractive index changes the two-lobe interference pattern of the fiber generates energy exchange. The relationship of the refractive index and the normalized value which is defined as difference to the sum of the two lobes interference intensity is studied. The analyzed results demonstrate that the sensor has refractive index sensitivity of 31.9/RIU (Normalized Value/ Refractive Index Unit) within the range of 1.36-1.4 RIU. As the two modes in the fiber have the same response to the temperature, the sensor possesses the anti-interference ability. Because of the easy fabrication, compact size and the ability of anti-interference to temperature make the sensor has extensive application prospects.
\end{abstract}

Keywords-refractive index sensor; polarization maintaining fiber; two-lobe interference; photonic crystal fiber; tapered fiber

\section{INTRODUCTION}

Fiber refractive index (RI) sensors have drawn great interest in recent years thanks to their compactness, high sensitivity, short response time, and anti-electromagnetic. Recently, refractometer based on optical tapered fiber[1-3], long-period fiber grating (LPFG) [4,5], and surface plasmon resonant (SPR) optical fiber [6-8] have been

\author{
Ailing Tan \\ College of Information Science and Engineering \\ Yanshan University \\ Qinhuangdao, P. R. China \\ The Key Laboratory for Special Fiber and Fiber Sensor \\ of Herbei Province \\ Yanshan University \\ Qinhuangdao, P. R. China \\ e-mail: tanailing@ysu.edu.cn \\ Qi Yuefeng \\ College of Information Science and Engineering \\ Yanshan University \\ Qinhuangdao, P. R. China \\ The Key Laboratory for Special Fiber and Fiber Sensor \\ of Herbei Province \\ Yanshan University \\ Qinhuangdao, P.R.China \\ e-mail: yfqi@ysu.edu.cn
}

widely investigated. However, optical fiber grating sensor needs expensive spectrometer for sensor detection. And the tapered fiber is sensitive to the external temperature. The SPR fiber needs complicated fabrication techniques. In order to overcome the above shortcomings, a tapered polarization maintaining photonic crystal fiber refractive index sensor is created by means of cladding corrosion.

In previous reports, the research on intermodal interference characteristics of the polarization maintaining fiber has been focused on the $L P_{01}^{x}$ and $L P_{01}^{y}$ based on the sagnac effect [9-11]. And the transmission spectrum needs complicate demodulation system. But the dual-mode interference between $L P_{01}^{x}$ and $L P_{11}^{x}$ modes is rarely used to refractive index detection. When the external refractive index changes, the two-lobe interference pattern which generated by the two basic modes in $x$ direction alters correspondingly. The normalized value of the difference to sum of the two-lobe energy indicates the liquid refractive index.

In this letter, the intermodal interference of $L P_{01}^{x}$ and $L P_{11}^{x}$ modes in tapered polarization maintaining photonic crystal fiber (PM-PCF) is investigated and adopted to the design of refractive index sensor. The mode propagation characteristics are calculated, and the interference output intensity patterns in the fiber are illustrated. Furthermore, the relationship between refractive index and the normalized value of the two-lobe interference intensity is analyzed. The sensor exhibits the sensitivity of $31.9 /$ RIU 
within the range of 1.36-1.4 RIU. Because of the same response to the temperature, it possesses high temperature stability.

\section{THEORETICALBACKGROUND}

The PM-1550-01 polarization maintaining photonic crystal fiber is purchased from Crystal Fiber A/S Inc. The microscope image and schematic diagram of the fiber is shown in Fig.1(a) and (b).

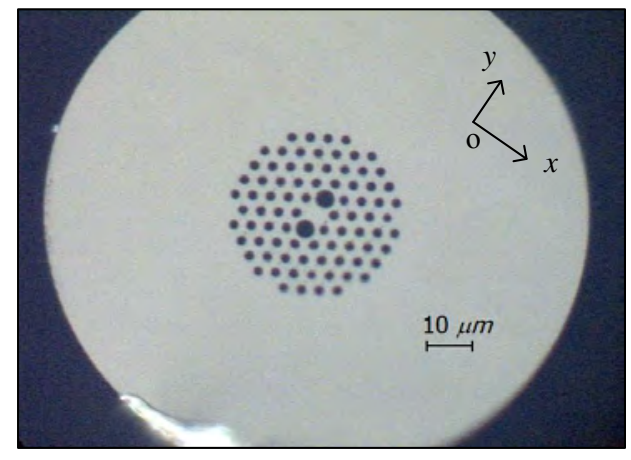

(a) Microscope image

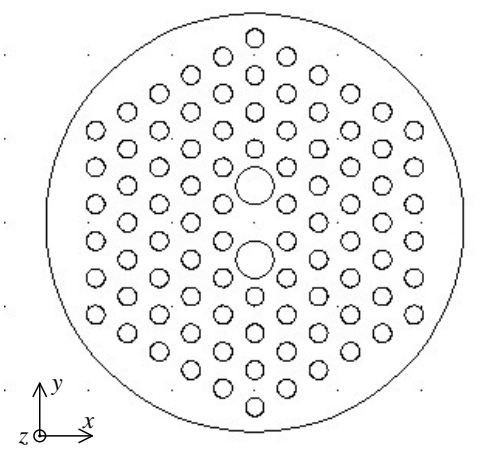

(b) Schematic diagram

Figure 1. The graph of PM-PCF

The outer holes diameter is $4.5 \mu \mathrm{m}$, and the inner big holes' is $2.2 \mu \mathrm{m}$. The hole to hole pitch $\Lambda$ is $4.4 \mu \mathrm{m}$. The background material is pure silica, and the Sellmeier equation is used for calculating the chromatic dispersion of silica. The cladding holes are filled with air $n_{\text {air }}=1.0$. Because of the two big holes, the symmetrical structure is disturbed. As a result, the modes in the fiber exhibit polarization maintaining characteristic. For example, there are four polarization modes exist at $1000 \mathrm{~nm}$ as is shown in Fig.2.

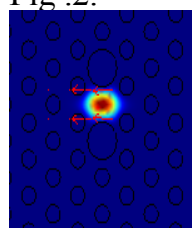

(a) $L P_{01}^{x}$

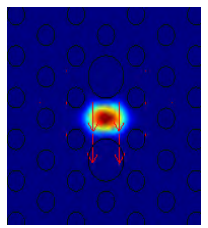

(b) $L P_{01}^{y}$

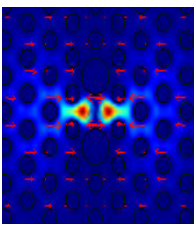

(c) $L P_{11}^{x}$

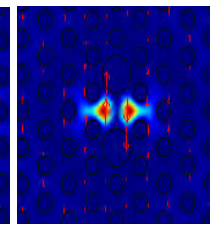

(d) $L P_{11}^{y}$
Figure 2. The distribution of the mode patten at $1000 \mathrm{~nm}$

In order to obtain the interference between two modes and generate two-lobe interference pattern, the modes in $x$ orientation ( $L P_{01}^{x}$ and $L P_{11}^{x}$ modes) are kept. However, the modes in the other orientation are eliminated by optical fiber polarimeter. If the $L P_{01}^{x}$ and $L P_{11}^{x}$ modes are excited by the light source simultaneously, the two-lobe interference patten will be exhibited on the output facet of the PM-PCF. The schematic diagram of two polarization maintaining modes interference is shown in Fig .3.

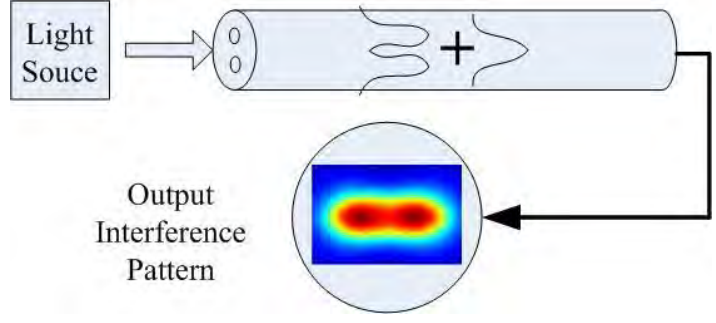

Figure 3. The schematic diagram of two modes interference

The output $x$-oriented interference intensity of the $L P_{01}^{x}$ and $L P_{11}^{x}$ modes can be expressed as

$$
\begin{aligned}
I(r, \varphi, z) & =|E(r, \varphi, z)|^{2} \\
& =\left|E_{L P_{01}^{x}}(r, \varphi, z)+E_{L P_{11}^{x}}(r, \varphi, z)\right|^{2} \\
& =E_{L P_{01}^{x}}(r, \varphi, z)^{2}+E_{L P_{11}^{x}}(r, \varphi, z)^{2} \\
& +2 E_{L P_{01}^{x}}(r, \varphi, z) E_{L P_{11}^{x}}(r, \varphi, z)^{2} \cos (\Delta \varphi)
\end{aligned}
$$

Where $E_{L P_{01}^{x}}(r, \varphi, z)$ is the transverse vector of the electric field of the optical fiber. $\Delta \varphi$ is the phase difference between the $L P_{01}^{x}$ and $L P_{11}^{x}$ modes, and can be expressed as:

$$
\Delta \varphi=\Delta \beta \cdot L
$$

Where $\Delta \beta$ denotes the propagation constant difference between the $L P_{01}^{x}$ and $L P_{11}^{x}$ modes, and $L$ is the optical fiber length. Equation (1) indicates that the output light intensity varied considerably with $\Delta \varphi$. When $\Delta \varphi$ changes from 0 to $2 \pi$, the output intensity varies correspondingly. The output intensity patterns are illustrated in Fig .3.
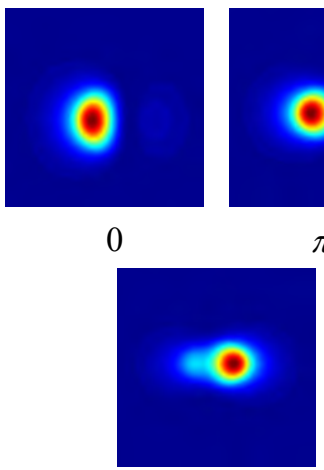

$2 \pi / 3$

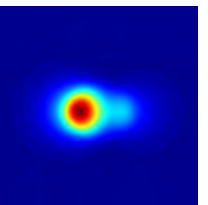

$\pi / 3$

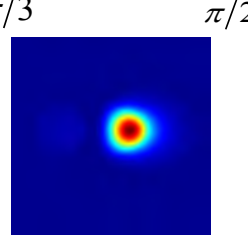

$\pi$
Figure 4. The output intensity energy with different $\Delta \varphi$ 
In this case, if the intensity of the two lobes are monitored, the phase difference will be obtained. And the external disturbance which causes the phase difference shift can be measured.

\section{ANALYSE OF RI SENSING USING TAPERED PM-PCF}

In order to enhance the RI sensitivity, the PM-PCF is tapered by hydrofluoric acid corrosion. The sensing system diagram is illustrated in Fig .5. In the system, the optical fiber polarization is used to obtain $\mathrm{X}$-oriented modes. The two-lobe intensity is captured by the side lobe extractor.

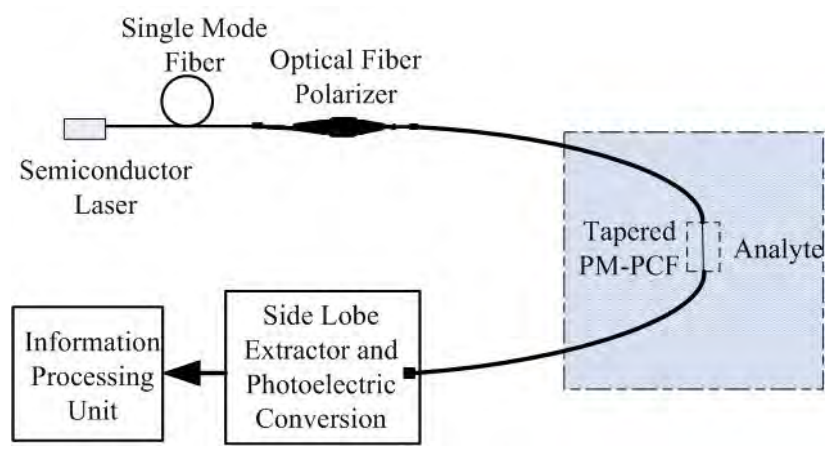

Figure 5. The RI sensing system diagram

The external analyte refractive index will affect the effective index of the PM-PCF. The relation of the effective index and the propagation constant difference is determined by

$$
\Delta \beta=\left(\beta_{01}-\beta_{11}\right)=\left(n_{\text {eff } 01}-n_{\text {eff } 11}\right) \cdot 2 \pi / \lambda
$$

Where $n_{\text {eff } 01}$ and $n_{\text {eff } 11}$ is the effective index of the $L P_{01}^{x}$ and $L P_{11}^{x}$ modes.

\section{A. The Relationship between RI and the Normalized value of Two-lobe Interference Intensity}

The external refractive index has effect on the effective index of the transmission modes. The finite element method is adopted for calculating the effective index of $L P_{01}^{x}$ and $L P_{11}^{x}$ modes. When the wavelength is $1000 \mathrm{~nm}$ and the radius of the fiber is chosen $25 \mu \mathrm{m}$, the effective index is analyzed. The results show that the basic mode $L P_{01}^{x}$ keeps the same value as the analyte refractive index increases. However, the second mode $L P_{11}^{x}$ increases correspondingly. The effective index difference between $L P_{01}^{x}$ and $L P_{11}^{x}$ modes is shown in Fig .6.

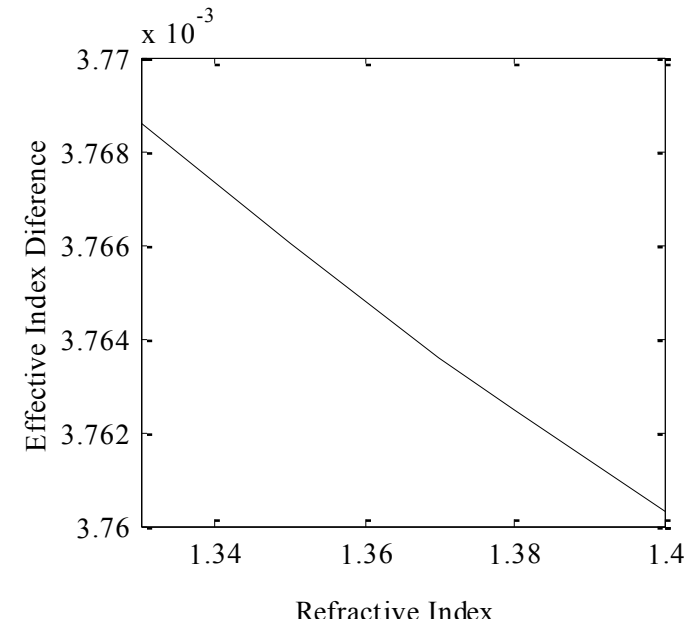

Figure 6. The effective index difference of the two modes with different analyte refractive index

As a result, the parameter $\Delta \beta$ will decreases as the analyte RI changes. The output interference intensity depends on $I(r, \varphi, z)$ calculated by Equation (1). The refractive index sensing characteristic of the PM-PCF is verified in Fig .7.

The tapered part of the PM-PCF sensor is $2 \mathrm{~cm}$ long. From Fig .7, it can be seen that the two-lobe energy exchanges as the analyte RI increase. When the RI increases from 1.33-1.35 the left lobe intensity is enhanced gradually. However, the right lobe intensity increases obviously as the RI changes from 1.37 to 1.4 . The key point of the work is to get the two-lobe energy intensity and determine the radio of them. Furthermore, the refractive index of the analyte can be detected.

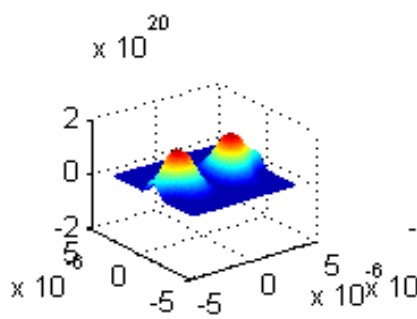

$$
\times 10^{20}
$$

$$
\times 10^{20} \quad \text { (a) } 1.33
$$

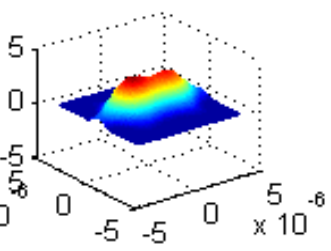

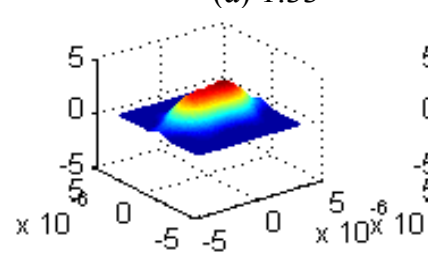

(c) 1.37
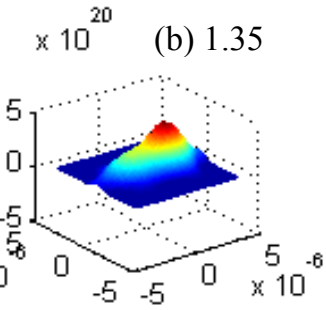

(d) 1.4
Figure 7. The two-lobe inference pattern with $1.33,1.35,1.37$ and 1.4

$$
\text { RIU }
$$

The normalized value is defined as the difference to the sum of the two-lobe intensity, which is shown as:

$$
N=\frac{I_{\text {Right }}-I_{\text {Left }}}{I_{\text {Right }}+I_{\text {Left }}}
$$


The value indicates the output intensity energy exchange of the two lobes. Therefore the relation between the normalized value and the analyte refractive index is studied, which is illustrated in Fig .8.

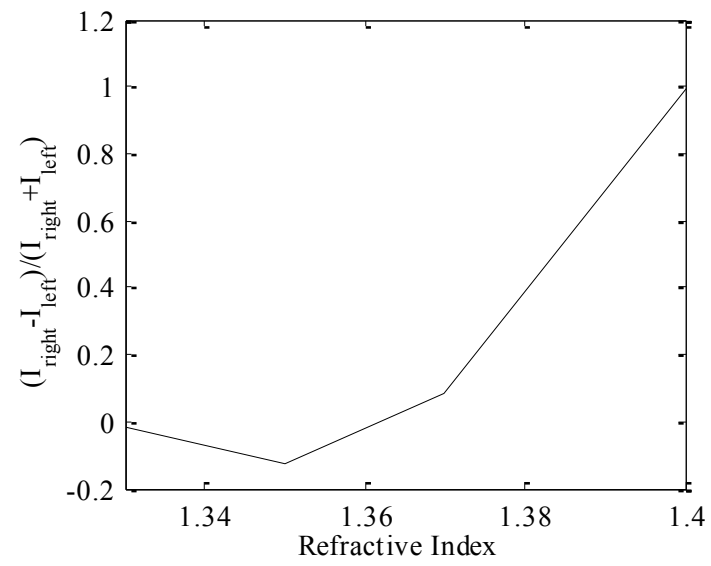

Figure 8 . The normalized value with different RI

From Fig .8 we can find that the normalized value reduces as the refractive index increase from 1.33-1.35. However, the normalized value goes up within 1.35-1.4. And the higher the refractive index is the faster the slope rate grows. It is obviously that the sensor is more sensitive to the high refractive index around 1.4. Then the sensitivity is researched as follows:

$$
\Delta N=\frac{\partial N}{\partial n} \cdot \Delta n
$$

Where the parameter $N$ is the normalized value we defined, and $n$ is the analyte refractive index value. The parameter $\frac{\partial N}{\partial n}$ indicates the refractive index sensitivity of the tapered PM-PCF sensor. When the wavelength is chosen $1000 \mathrm{~nm}$, and the tapered part length is $2 \mathrm{~cm}$, the sensitivity is around 31.9/RIU (Normalized Value/Refractive Index Unit). More importantly, the light intensity demodulation system is simpler and cheaper than the spectrum demodulation system which is used in the optical fiber grating system.

\section{B. The Influecn of the Temperature}

In order to investigate the temperature characteristic of the refractive index sensor, we consider both of the thermal expandability and thermo-optic effect on the polarization maintaining photonic crystal fiber. The young's modulus of silica is $E=73.1 \times 10^{9}$, Poission's ratio of it is $v=0.17$, and the thermal expansion coefficient is $\alpha=5 \times 10^{-7}$. Supposing the thermo-optic coefficient of silica core is $\xi^{c o}=8.5 \times 10^{-6} /{ }^{\circ} \mathrm{C}[13]$, the relationship between the effective index difference of the $L P_{01}^{x}$ and $L P_{11}^{x}$ modes and the temperature is shown in Fig .9.

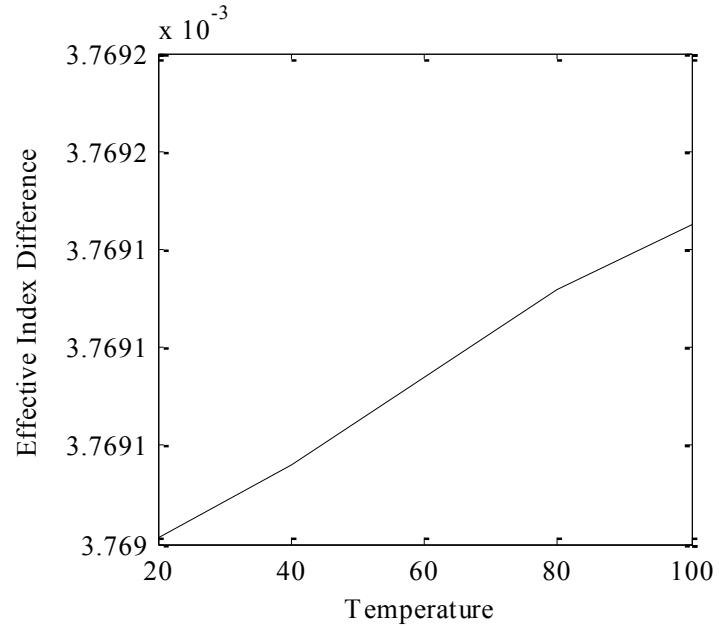

Figure 9. The relation between temperature and effective index

$$
\text { difference of } L P_{01}^{x} \text { and } L P_{11}^{x} \text { modes }
$$

In Fig .9, the effective index difference changes slightly as the temperature increases. Therefore, the PMPCF RI sensor has anti-interference ability to the temperature.

\section{CONCLUSION}

In this letter, a design of tapered PM-PCF is proposed to detect the refractive index. The refractive index characteristic of the sensor is numerically analyzed based on the finite element method. The analyzed results show that refractive index sensitivity is around 31.9/RIU within the range of 1.36-1.4 RIU. Although the polarization modes are sensitive to the analyte refractive index respectively, they respond similarly to the temperature disturbance. So the normalized value of the output twolobe interference intensity will eliminate the temperature disturbance to get high stability. Therefore the RI sensor we proposed has the anti-interference ability to the temperature. Moreover the best advantage of the senor is the simplified light demodulation system which can be used to the real-time monitor site.

\section{ACKNOWLEDGMENT}

The authors are grateful to the support of Herbei Provincial Science \& Technology Research and Development Project (No.13273305, No.12273302), and the support of Herbei Provincial Natural Science Foundation (No. F2013203252). The authors also thank the support of Yanshan University Doctor Fundation (No.B872) and National Natural Science Foundation of China (No. 61275093).

\section{REFERENCES}

[1] P.F. Wang, G. Brambilla, M.Ding, Y. Semenova, Q. Wu, G. Farrell,"High Sensitivity Evanescent Field Refractometric Sensor Based on a Ttapered Multimode Fiber Interference" Optics Letters, Vol. 36 June, 2011, pp. 2233-2235 DOI: 10.1364/OL.36.002233

[2] W. B.Ji, H.H. Liu, S.C. Tjin, K.K. Chow, A. Li. "Ultrahigh Sensitivity Refractive Index Sensor Based on Optical Microfiber", IEEE, Photonics Technology Lett. Vol. 24, Oct. 2012, pp.18721874, DOI: 10.1109/LPT.2012.2217738 
[3] Rui Yang, Yong-Sen Yu, Chao Chen, Yang Xue, and et al. "STapered Fiber Sensors for Highly Sensitive Measurement of Refractive Index and Axial Strain'IEEE Aerospace and Electron System Society. Vol. 30, Oct. 2012, pp.3126-3132, DOI: 10.1109/JLT.2012.2210997

[4] Guo Xuan, Bi Weihong, Liu Feng. "Refractive index sensing characteristics of fiber bragg grating in the polarization maintaining microstructured optical fiber "Proc. SPIE, SPIE in press, Vol. 8199, Nov. 2011, pp.8199v1-10, DOI: $10.1117 / 12.904702$

[5] L. Coelho, D. Viegas, J.L. Santos, J.M.M.M.de Almeida. "Enhanced refractive index sensing characteristics of optical fibre long period grating coated with titanium dioxide thin films" Sensors and Actuators B, Vol. 202, Oct. 2014, pp.929-934, DOI: 10.1016/j.snb.2014.06.035

[6] M.H. Tu, T. Sun, K.T.V. Grattan. "Optimization of goldnanoparticle-based optical fibre surface plasmon resonance (SPR)based sensors" Sensors and Actuators B: Chemical, Vol. 164, Mar. 2012, pp, 43-53, DOI: 10.1016/j.snb.2012.01.060

[7] Sarika Singh, Satyendra K. Mishra, Banshi D. Gupta. "Sensitivity enhancement of a surface plasmon resonance based fibre optic refractive index sensor utilizing an additional layer of oxides" Sensors and Actuators A: Physical, Vol. 193, Apr.2013, pp. 136140, DOI: $10.1016 /$ j.sna.2013.01.012
[8] Wei Wei, Xia Zhang, Xin Guo, Long Zheng, and et al. "Refractive index sensors based on Ag-metalized nanolayer in microstructured optical fibers" Optik-International Journal for Light and Electron Optics, Vol. 123, July. 2012, pp.1167-1170, DOI: 10.1016/j.ijleo.2011.07.044

[9] K.S. Lima, C.H. Puaa, S.W. Haruna, b, H. Ahmada. "Temperature-sensitive dual-segment polarization maintaining fiber Sagnac loop mirror", Optics \& Laser Technology, Vol. 42, Mar. 2010, pp. 377-381, DOI: 10.1016/j.optlastec.2009.08.015

[10] Lihui Wanga, Jianfei Jib, Qiangsheng Bob, Yubo Yuanb, Jiabin Qiana. "Modeling and simulation of polarization errors in Sagnac fiber optic current sensor" Optik-International Journal for Light and Electron Optics, Vol. 125, Sep. 2014, pp. 4770-4775, DOI 10.1016/j.ijleo.2014.04.064

[11] Houhui Liang, Mingming Sun, Yongxing Jin. "Twist sensor based on Sagnac single-mode optic fiber interferometer", Optik International Journal for Light and Electron Optics, Vol. 124, Dec. 2013, pp. 6676-6678, DOI: 10.1016/j.ijleo.2013.05.105

[12] Guan Shouhua, Yu Qing-xu, Song Shide, and Zheng Jianzhou, "Theoretical and experimental study on temperature characteristic of long-period fiber grating" Chinese Journal of Sensor and Actua Actuators, Vol. 20, Mar. 2007, pp. 543-545, EEACC : 7230 E 